\title{
ÉTUDE D'UN FILTRE ACTIF A TRANSISTORS ASSOCIÉ A UN REDRESSEUR POLYPHASÉ DE GRANDE PUISSANCE FONCTIONNANT EN RÉGIME D'IMPULSIONS
}

\author{
R. PRAJOUX, G. JUANOLE \\ Laboratoire d'Automatique et de ses Applications Spatiales \\ du C. N. R. S.-B. P. 4036, 31, Toulouse, France
}

(Reçu le 16 décembre 1969, révisé le 16 mars 1970)

\begin{abstract}
Résumé. - Cet article concerne la régulation d'impulsions de courant de longue durée (quelques $100 \mathrm{~ms}$ ), produites par des générateurs utilisant un redresseur polyphasé, et destinées à des aimants de grande puissance utilisés en Physique pour l'obtention de champs magnétiques de grande énergie et de grande stabilité.

Les auteurs traitent des systèmes à hautes performances comprenant un filtre actif de puissance et abordent les principaux problèmes relatifs à de tels ensembles dans le but d'en effectuer la synthèse.

Abstract. - This paper is concerned with the regulation of long duration (some $100 \mathrm{~ms}$ ) current impulses produced by generators using a polyphase rectifier, for high power magnets used in Physics to obtain high energy and high stability magnetic fields.

The authors deal with high performance systems including an active power filter and study the principal problems encountered in such systems, in order to make their synthesis.
\end{abstract}

Introduction. - La présente étude concerne les alimentations de grande puissance fonctionnant par conversion d'un réseau alternatif au moyen de redresseurs polyphasés. Ces alimentations sont en général destinées à la production de champs magnétiques.

Les redresseurs présentent, par leur fonctionnement discontinu, un signal de sortie contenant des composantes alternatives harmoniques d'amplitude notable. L'obtention d'un champ magnétique sans ondulation résiduelle implique un filtrage du signal de sortie du redresseur.

Une catégorie importante de dispositifs utilisés est constituée par les systèmes fonctionnant en impulsions, ceci dans le but de faciliter le dimensionnement de l'appareillage de puissance. Le temps d'établissement du champ magnétique devant être inférieur à une limite donnée, généralement de l'ordre de la constante de temps de l'aimant, on ne peut utiliser un filtre passif seul. On adjoint donc au redresseur un organe jouant le rôle d'un filtre mais pouvant être commandé : cet organe est appelé un filtre actif.

Nous nous intéresserons ici uniquement aux filtres actifs destinés à la production de tels champs magnétiques pulsés.

Les travaux du Laboratoire d'Automatique et de ses Applications Spatiales du Centre National de la Recherche Scientifique dans ce domaine ont débuté par l'étude d'un filtre actif destiné à fonctionner avec un redresseur polyphasé à 48 phases $(60 \mathrm{~V}, 16 \mathrm{kA})$. Cet ensemble devait alimenter un des aimants d'éjection du faisceau du synchro-cyclotron du C. E. R. N. à
Genève et fonctionne comme tel depuis $1965[9,10]$. Actuellement l'étude d'un filtre actif destiné à l'alimentation principale du synchro-cyclotron Saturne de Saclay est en cours.

Les paragraphes qui vont suivre constituent un résumé des principaux résultats obtenus au cours de ces travaux.

I. Généralités sur un ensemble redresseur et filtre actif. - I. 1 SCHÉMA DE PRINCIPE DE L'ENSEMBLE (Fig. I.1). - L'ensemble se présente conformément à la figure $\mathrm{I}$. 1. Le filtrage étant obtenu par une régulation, il existe une tension de référence $V_{c}$ qui commande l'asservissement et un organe capteur (shunt si la grandeur à réguler est le courant). Il peut arriver que l'on s'intéresse à la dérivée $\mathrm{d} B / \mathrm{d} t \mathrm{du}$ champ magné-

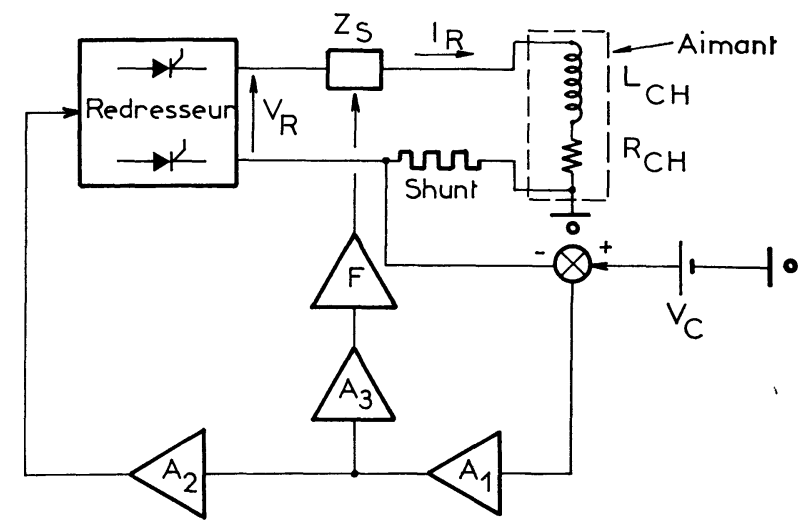

Fig. I.1. - Schéma de principe du système. 
tique plutôt qu'au champ $B$ lui-même. On est alors conduit à réguler la tension aux bornes de l'aimant et l'organe capteur est un pont diviseur résistif. Les amplificateurs A1, A2, A3 traitent le signal d'erreur et commandent le redresseur polyphasé et le filtre actif $\mathrm{F}$. $Z_{s}$ est l'organe de transmission de la puissance du filtre dans le circuit principal.

I. 2 HYPOTHÈSE SUR LE COMPORTEMENT DU REDRESSEUR. - Un redresseur polyphasé est un organe de nature essentiellement non linéaire. Son utilisation en tant qu'amplificateur de puissance dans une boucle de commande pose de nombreux problèmes théoriques.

Dans ce qui suit nous supposerons que le redresseur se comporte, pour les petits signaux, c'est-à-dire pour des petites variations de sa tension de commande, comme un amplificateur parfait de gain $K$ à la sortie duquel s'ajoute une source de tension $\mathcal{V}_{R}(t)$ représentant l'ondulation résiduelle (cf. Fig. I.2).

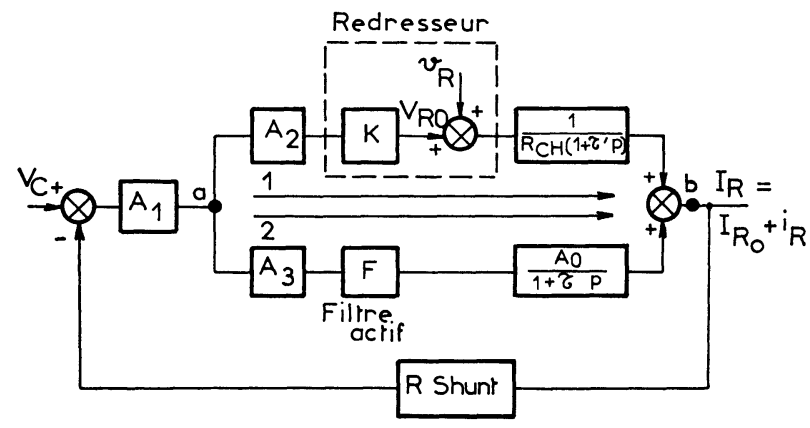

Fig. I.2. - Schéma bloc du système.

Cette représentation est valable en $1^{\text {re }}$ approximation, pour un point de fonctionnement donné, et lorsque la fréquence des signaux de commande est faible devant la fréquence d'échantillonnage du redresseur. Cette représentation sera suffisante pour ce que nous traitons au paragraphe suivant.

Cependant, l'étude rigoureuse du comportement d'une boucle de commande comprenant un redresseur polyphasé, étude très importante pour une bonne compréhension des systèmes qui nous occupent ici, est en cours actuellement au L. A. A. S. [6, 7, 8].

I.3 SCHÉMA BLOC (Fig. I.2). - Bien que nous ne connaissions pas a priori la transmittance exacte qui lie le courant dans la charge au courant de sortie du filtre, nous pouvons considérer qu'elle sera de la forme suivante :

$$
\frac{I_{R}(p)}{I_{f}(p)}=\frac{A_{0}}{1+\tau p}
$$

$I_{R}(p)$ transformée de Laplace du courant dans la charge,

$I_{f}(p)$ transformée de Laplace du courant fourni par le filtre,

$f=(1 / 2 \pi \tau)$ fréquence de coupure du filtre [2].
Par ailleurs

$$
\frac{I_{R}(p)}{V_{R}(p)}=\frac{1}{R_{\mathrm{CH}}\left(1+\tau^{\prime} p\right)}
$$

$\tau^{\prime}=\frac{L_{\mathrm{ch}}+L_{\mathrm{S}}}{R_{\mathrm{ch}}}=$ constante de temps totale de la charge (cf. paragraphe I.3).

La chaîne (1), dite chaîne lente, fournit la puissance continue.

La chaîne (2), dite chaîne rapide, fournit la puissance nécessaire à la compensation de l'ondulation.

La chaîne lente a une fonction de transfert du type passe bas. Au-delà d'une certaine fréquence $f_{s}$, son gain devient petit devant celui de la chaîne rapide et le schéma bloc pour les fréquences supérieures à $f_{s}$ est comme sur la figure I.3.

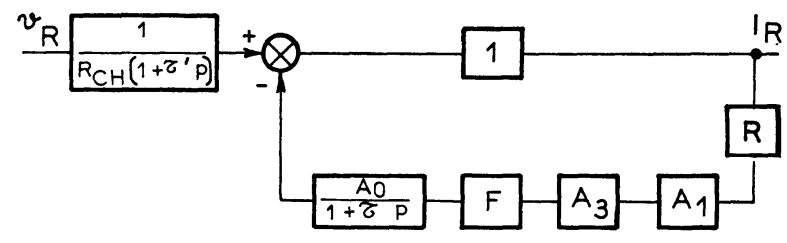

Fig. I.3. - Schéma bloc pour les fréquences supérieures à la fréquence $f_{s}$.

Le gain en boucle ouverte du système de la figure I. 3 est :

$$
G(p)=A_{1}(p) A_{2}(p) F(p) \frac{A_{0}}{1+\tau p} \cdot R
$$

Nous avons donc :

$$
I_{R}(p)=\frac{V_{R}(p)}{R_{\mathrm{ch}}\left(1+\tau^{\prime} p\right)} \cdot \frac{1}{1+G(p)} .
$$

La tension d'ondulation résiduelle $\mho_{R}(t)$ est une tension périodique, donc décomposable en série de Fourier. En notation complexe nous avons :

$$
\bigcup_{R}(t)=\sum_{i=1}^{n} \mho_{i} \mathrm{e}^{j\left(\omega_{i} t+\varphi_{i}\right)}
$$

Le courant d'ondulation $i_{R}(t)$ qui en résulte est de la forme :

$$
i_{R}(t)=\sum_{i=1}^{n} i_{i} \mathrm{e}^{j\left(\omega_{i} t+\psi_{i}\right)}
$$

D'après I.4 l'amplitude $i_{i}$ de l'ondulation en courant à la pulsation $\omega_{i}$ est donc :

$$
i_{i}=\frac{\vartheta_{i}}{\left|R_{\mathrm{ch}}\left(1+\tau^{\prime} j \omega_{i}\right)\right|} \cdot \frac{1}{\left|1+G\left(j \omega_{i}\right)\right|} .
$$

Le premier facteur du second membre représente l'amplitude de l'ondulation du courant $\left(i_{i}\right)_{B O}$ dans 
l'aimant en l'absence du filtrage. En appelant $I_{R O}$ le courant continu dans l'aimant, nous avons :

$$
\frac{i_{i}}{I_{R o}}=\frac{\left(i_{i}\right)_{B o}}{I_{R o}} \cdot \frac{1}{\left|1+G\left(J \omega_{i}\right)\right|} .
$$

Compte tenu de l'atténuation $\frac{\left(i_{i}\right)_{B O}}{I_{R o}} / \frac{i_{i}}{I_{R o}}$ voulue, on peut ainsi déterminer le gain de boucle $\mathrm{G}$ nécessaire. Si le redresseur est suivi d'un filtre passif, le terme $\mho_{R}(t)$ représente évidemment l'ondulation résiduelle après le filtrage passif.

I.4 SYSTÈME DE COMMANDE MIXTE. $-\bigcup_{R}(t)$ se présente comme une perturbation appliquée à l'asservissement. Nous avons vu au paragraphe précédent comment on peut, en agissant sur le gain de boucle $\mathrm{du}$ système, réduire l'ondulation en courant $i_{R}(t)$ provoquée par $\mathcal{V}_{R}(t)$. On peut par ailleurs envisager d'utiliser un système de commande mixte (cf. Fig. I.4) boucle ouverte - boucle fermée où l'on fait

$$
C(p)=1 / A(p) \text {. }
$$

La compensation en boucle ouverte se rattache à la théorie de l'invariance due à l'école d'Automatique de Kiev mais dont le principe est en fait connu depuis longtemps.

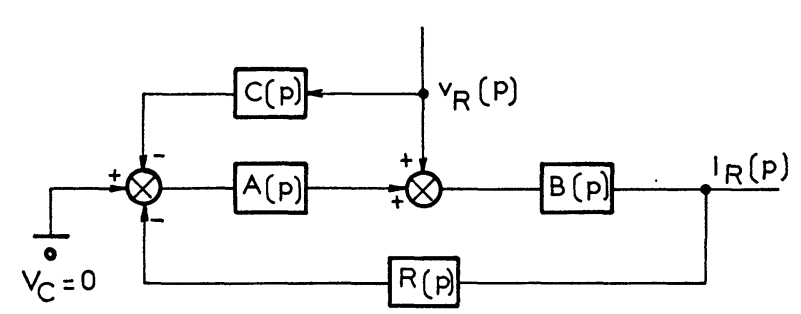

FIG. I.4. - Schéma bloc d'un système de commande mixte.

Une grande partie des difficultés d'application des systèmes de commande mixte provient de la mesure de la perturbation et de la réalisation de la transmittance $1 / A(p)$. Dans notre cas, la perturbation $V_{R}(t)$ est facilement mesurable. De plus, la transmittance $A(p)$ est celle d'un ensemble d'organes électroniques dont les transmittances propres peuvent être très bien connues. Pour ces deux raisons, l'utilisation de la compensation en boucle ouverte est ici réalisable.

De la figure I. 4 on tire :

$i_{R}(p)=\frac{B(p)}{1+A(p) B(p) R(p)} V_{R}(p)[1-C(p) A(p)]$.

Dans la mesure où les composantes du spectre de fréquence du signal $\mathfrak{L}^{-1}\left[\vartheta_{R}(p)[1-C(p) A(p)]\right]$ sont plus petites que celles du spectre de $\mho_{R}(t)$, on a besoin d'un gain de boucle plus faible pour la précision demandée.

D'une façon générale, ce type de commande est délicat à réaliser en pratique. Par ailleurs, la difficulté est encore plus grande dans les systèmes pulsés que dans les systèmes continus du fait des régimes transitoires qui résultent du mode de fonctionnement. Pour citer un exemple, la mesure de l'ondulation résiduelle de la tension de sortie du redresseur se fait en pratique en mesurant cette tension elle-même ; il en résulte qu'au terme $\mho_{R}(t)$ cherché s'ajoute une tension continue qui donnera lieu, à chaque impulsion, à un régime transitoire qui pourra être difficile à minimiser.

I. 5 StRUCtURE DU FILTRE ACTIF. - Le redresseur jouant le rôle de source de tension vis-à-vis de la charge le filtre doit, par le jeu de la boucle de régulation, ajouter à la tension du redresseur une tension $\mathcal{V}_{f}(t)$ destinée à compenser l'ondulation en tension $\mathcal{V}_{R}(t)$ du redresseur (cf. figure I.5). $\mho_{f}(t)$ doit être nulle en valeur moyenne afin que la circulation du courant continu dans le filtre n'entraîne pas une dissipation inutile de puissance.

Deux types de filtre peuvent être utilisés : le filtre parallèle et le filtre série. Le filtre parallèle présente des inconvénients quant à son utilisation dans les systèmes fonctionnant par impulsion.

Comme nous nous intéressons ici à ce type de fonctionnement, nous considérerons uniquement le cas du filtre série (Fig. I.5).

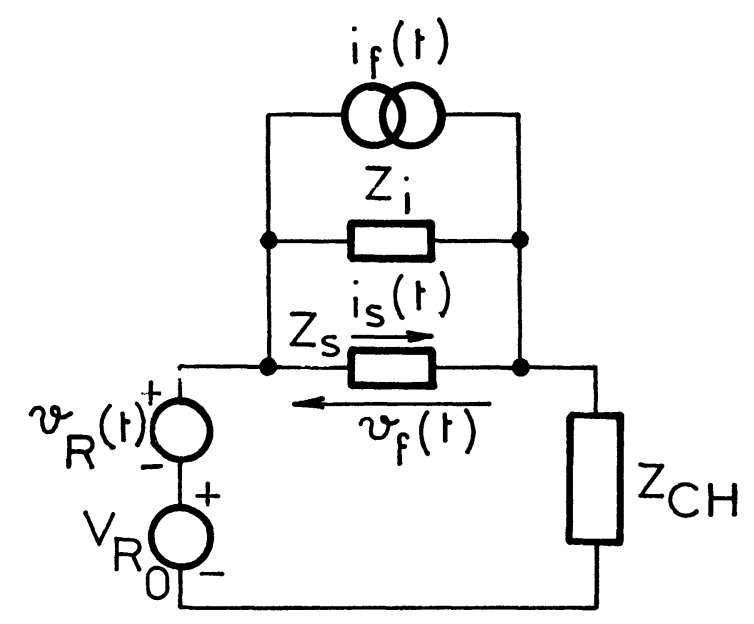

Fig. I.5. — Structure d'un filtre série.

La source de courant $i_{f}(t)$ d'impédance interne $Z_{i}$, débite dans l'impédance $Z_{s}$.

Le passage du courant continu $I_{R o}$ dans $Z_{s}$ ne devant pas entraîner de dissipation de puissance, $Z_{s}$ est un élément inductif. De plus, comme il faut adapter les caractéristiques du redresseur à celles de l'étage de puissance du filtre, $Z_{s}$ sera plus précisément un autotransformateur ou un transformateur, avec une préférence pour cette dernière solution qui procure en outre l'isolement de l'étage de puissance du filtre et du redresseur.

I. 6 Puissance DU Filtre. - Nous définissons la puissance du filtre comme la valeur maximum (compte tenu des caractéristiques limites de la source $i_{f}(t)$ 
réelle) du produit de la tension crête $V_{s}$ aux bornes du filtre par le courant crête $I_{s}$ fourni à l'inductance $L_{s}$ en régime sinusoïdal, le filtre actif étant à vide (cf. Fig. I.6).

$$
P_{\text {filtre }}=V_{s} \cdot I_{s}=L_{s}\left[\omega I_{s}^{2}(\omega)\right]_{M}
$$

$I_{s}(\omega)$ courant de crête maximum que le filtre peut fournir à $L_{s}$ à la pulsation $\omega$.

$\left[\omega I_{s}^{2}(\omega)\right]_{M}$ : valeur maximum du produit entre crochets.

$\boldsymbol{P}_{\text {filtre }}$ est une puissance apparente ; elle caractérise parfaitement la dimension du filtre et permet de comparer deux filtres de caractéristiques $V_{s}$ et $I_{s}$ différentes.

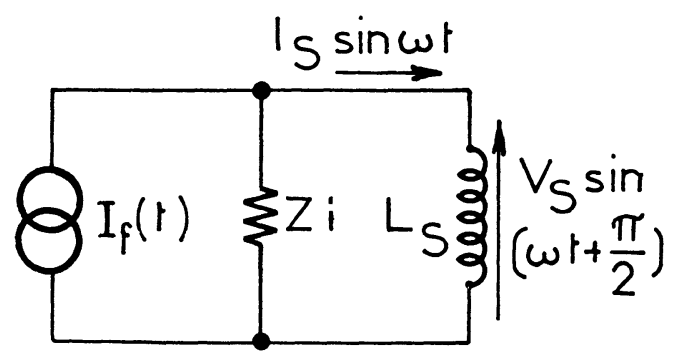

FIG. I.6. - Filtre actif, à vide, en régime sinusoïdal.

II. Etude de l'étage de puissance d'un filtre série. Le problème posé est de définir un étage de puissance comprenant un nombre minimum de composants tout en ayant une très bonne fiabilité.

Le choix du composant est fixé par la puissance demandée au filtre. Les tubes électroniques sont particulièrement intéressants pour les grandes puissances ; pour les faibles et moyennes puissances (de l'ordre de $10 \mathrm{~kW}$ ) on utilise généralement des transistors de puissance au silicium dont les avantages sont bien connus. Nous considérerons dans ce qui suit le cas d'un étage de puissance à transistors.

La fiabilité du système est surtout conditionnée par l'apparition du phénomène de $2^{e}$ disruption [3-4-5].

C'est dans cette optique que nous allons étudier un étage de puissance répondant aux exigences ci-dessus.

II. 1 PhÉNOMÈNE DE DEUXIÈME DISRUPTION - La puissance électrique $P_{c}$ fournie à un transistor est dissipée en pratique dans la jonction collecteur-base. La génération de chaleur dans cette zone active s'accompagne d'une modification des paramètres électriques. Cette modification peut conduire soit à un accroissement global de la puissance appliquée au niveau de la jonction collecteur-base soit à l'instabilité de la distribution initiale de la puissance.

Le premier phénomène est l'échauffement cumulatif global, phénomène bien connu dont il est clair que l'on doit s'affranchir par un circuit de commande adéquat du transistor de puissance.

Le second phénomène appelé instabilité thermique latérale est favorisé par la présence d'un courant base inverse, par le fonctionnement en première avalanche et par un courant base élevé. De plus, le phénomène de deuxième disruption peut apparaître par échauffement global lorsque la jonction collecteur base atteint une valeur critique de la température [2].

II. 2 ReMARQUE FONDAMENTALE SUR LE FONCTIONNEMENT DU FILTRE. - Examinons le fonctionnement de l'ensemble lorsque la régulation agit, c'est-à-dire lorsqu'il y a très peu d'ondulation aux bornes de la charge. La tension $\mathcal{U}_{f}(t)$ introduite par le filtre dans le circuit principal est constamment opposée à la tension d'ondulation $\bigcup_{R}(t)$ du redresseur (cf. figure I.5). Tout se passe donc comme si le filtre travaillait à vide.

Pour tous les calculs de ce chapitre on considérera le transformateur de transmission de la puissance du filtre dans le circuit principal comme fonctionnant à vide.

II.3 EtAGe DE PUISSANCE. - II.3.1 Structure. Nous choisissons un étage classe B dont les avantages vis-à-vis de la dissipation de puissance des transistors sont bien connus. Etant donné que nous utilisons un transformateur de sortie, nous pouvons adopter un étage symétrique (cf. Fig. II.1).

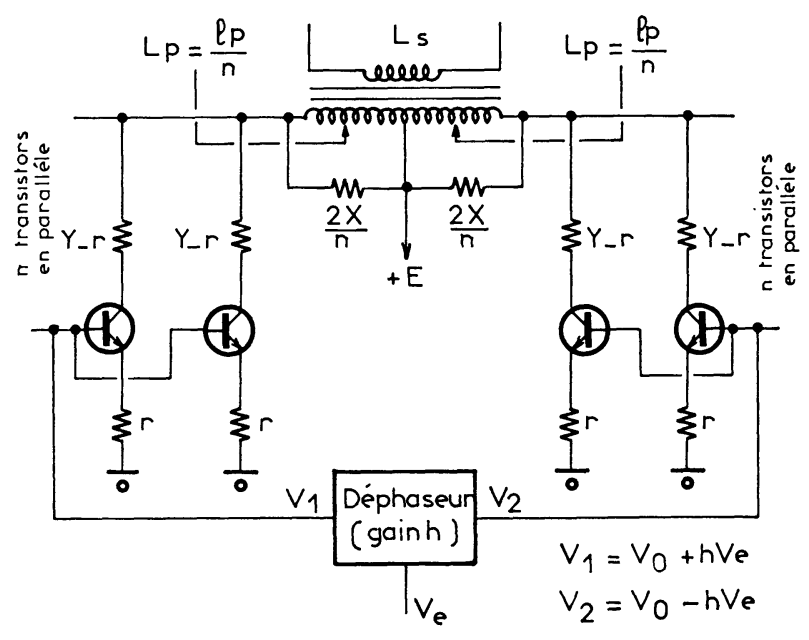

FIG. II.1. - Etage de puissance.

La présence de l'élément inductif $L_{p}$ dans le circuit collecteur des transistors conduit à l'utilisation des résistances $X$. Les résistances $Y$ limitent le courant maximum débité par les transistors et diminuent la puissance dissipée aux basses fréquences. Les résistances $r$ sont les résistances d'équilibrage des courants dans les transistors.

\section{Remarque :}

En supposant le couplage magnétique parfait entre les deux demi-primaires la moitié de l'étage qui conduit voit une résistance $X / n$ en parallèle sur son primaire.

II.3.2 Domaine de fonctionnement. - Considérons le montage relatif à un transistor d'une moitié de l'étage de puissance (cf. Fig. II.2). Nous considérerons dans ce qui suit que l'étage de puissance a un courant 
de repos négligeable et que la tension de saturation collecteur-émetteur est très petite.

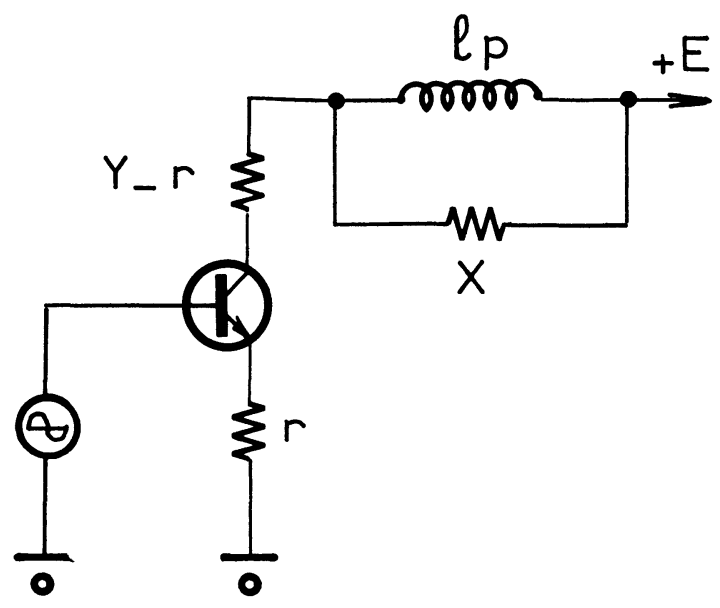

FIG. II.2. - Demi-étage ramené à un transistor.

Dans le plan $I_{c}, V_{C E}$ nous avons le système de droites de la figure II.3. La droite (1) est la droite de charge en continu du transistor. Supposons que le courant $I_{M}$ soit établi et que nous coupions brusquement la commande : le point de fonctionnement évolue suivant la droite (2). Ce mode de fonctionnement est celui où les plus grandes tensions collecteur-émetteur et les plus grandes puissances instantanées sont atteintes.

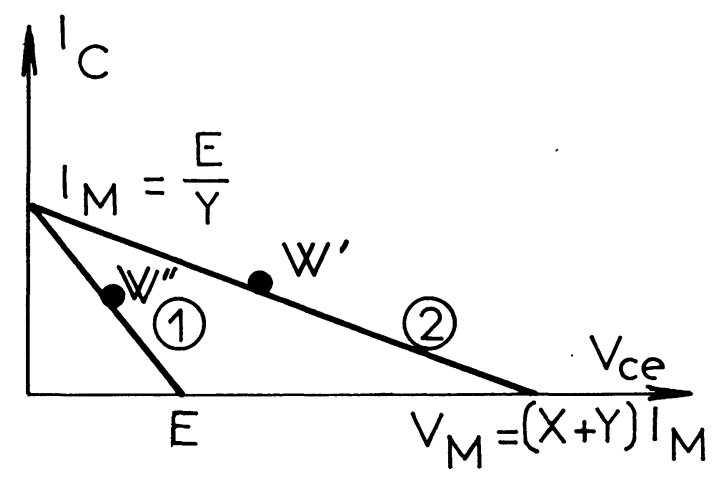

FIG. II.3. - Droites de charge relatives au montage de la figure II. 2.

Etant donné le paragraphe II. 1, pour ne pas favoriser l'apparition $\mathrm{du}$ phénomène de $2^{\mathrm{e}}$ disruption, le domaine de fonctionnement du transistor doit se trouver dans la zone non hachurée de la figure II.4.

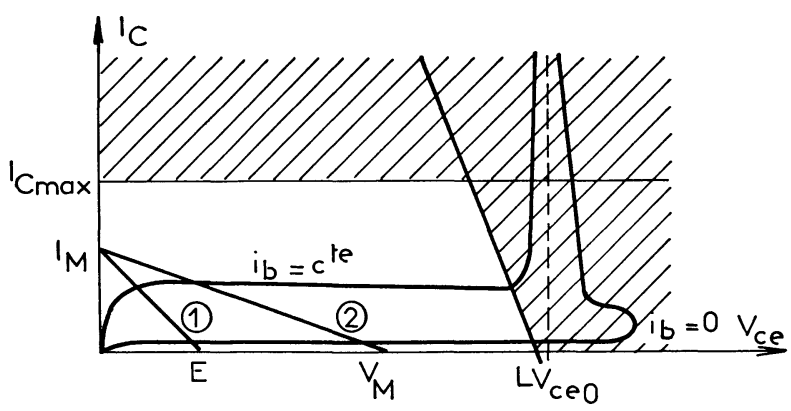

FIG. II.4. - Domaine de fonctionnement d'un transistor.
Les paramètres $E, X, Y$ seront donc tels que la droite (2) se trouve dans la zone permise.

De plus, on s'assurera évidemment qu'au cours d'un tel fonctionnement la température qu'atteint la jonction reste inférieure à la limite permise [2].

IJ.3.3 Dérive du point de repos. - Dans le montage de la figure II.1, la dérive des tensions base émetteur des transistors (qui peut atteindre plusieurs $100 \mathrm{mV}$ ) se superpose au signal de commande fourni par le déphaseur ce qui entraîne une dérive importante du point de fonctionnement de l'étage. Le fonctionnement en classe $B$ rendant très difficile la mesure du courant de repos, on ne peut stabiliser celui-ci par contreréaction. On peut alors adopter la configuration de la figure II. 5 .

$I_{1}$ : courant délivré par un côté de l'étage de puissance,

$I_{2}$ : courant délivré par l'autre côté de l'étage de puissance.

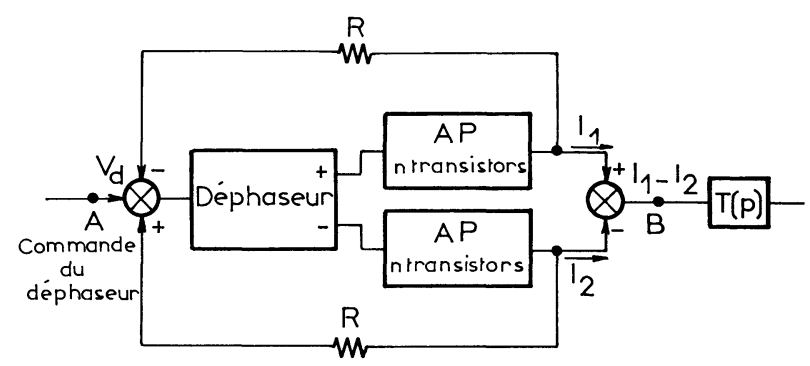

Fig. II.5. - Méthode de bouclage de l'amplificateur de puissance.

On réalise donc l'asservissement de $R\left(I_{1}-I_{2}\right)$ à $V_{d}$.

Un tel système n'empêche évidemment pas la dérive du point de repos mais permet de s'affranchir de ses conséquences. Lorsque le signal $V_{d}$ est tel que un côté de l'étage ne débite plus, par exemple $I_{2}=0$, on asservit le courant $I_{1}$ débité par l'autre côté à $V_{d}$ et l'on voit que la valeur maximum de $I_{1}$ ne dépend que de $V_{d}$ maximum.

Les courants $I_{1}$ et $I_{2}$ en fonction de $V_{d}$ sont comme sur la figure II. 6 où on a représenté 2 points de repos différents $I_{01}$ et $I_{02}$.

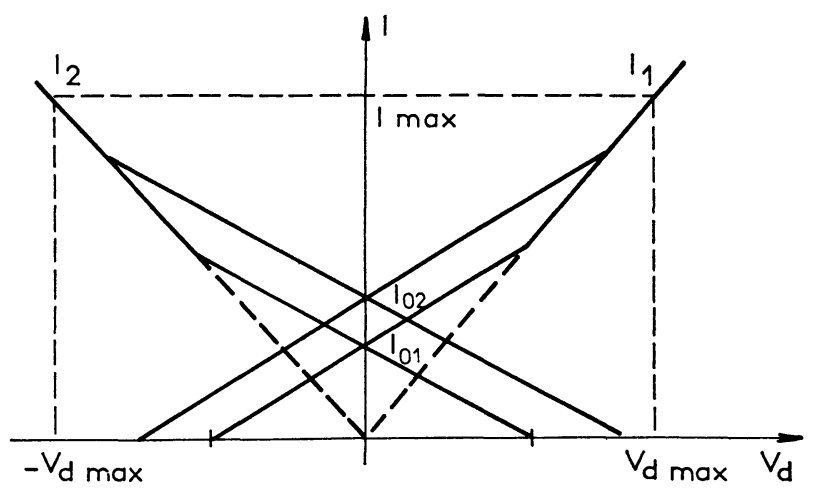

FIG. II.6. - Dérive du point de repos. 
Un avantage de la configuration précédente est que le gain entre $\mathbf{A}$ et $\mathbf{B}$ ne dépend pas du point de fonctionnement. Par suite l'élaboration de la boucle de commande principale s'en trouve facilitée.

II.4 Puissance De L'ÉtAge. - II.4.1 Fonctionnement maximal de l'étage. - Supposons que nous excitions sinusoïdalement les bases des transistors et que nous soyions en régime permanent. L'étage est à ses possibilités maximales lorsque les transistors sont à la limite de la saturation. Vis-à-vis de l'inductance $L_{p}$, à une pulsation donnée, on est alors dans les conditions de la figure II.7. Dans l'inductance $L_{p}$ circule un courant $I_{p}(\omega)$ tel que :

$$
I(\omega)=\left|I_{p}(\omega)\right|=\frac{n I_{M}}{\sqrt{1+\frac{\omega^{2}}{\omega_{c}^{2}}}}
$$

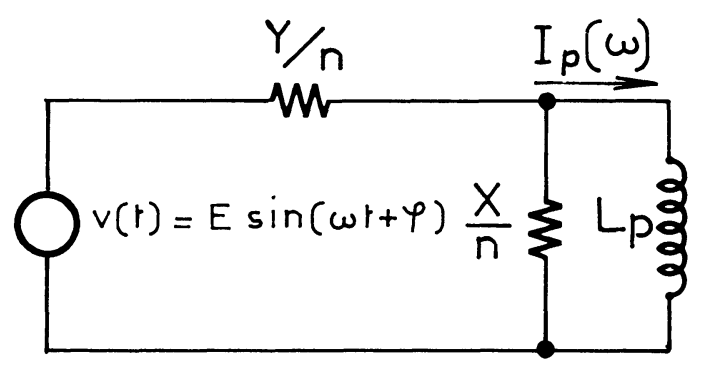

FiG. II.7. - Etage de puissance en régime sinusoïdal.

avec

$$
\omega_{c}=\frac{1}{l_{p}} \cdot \frac{X Y}{X+Y}=\frac{X Y}{n L_{p}(X+Y)} .
$$

La courbe $I(\omega)$ se présente en coordonnées logarithmiques comme sur la figure II.8.

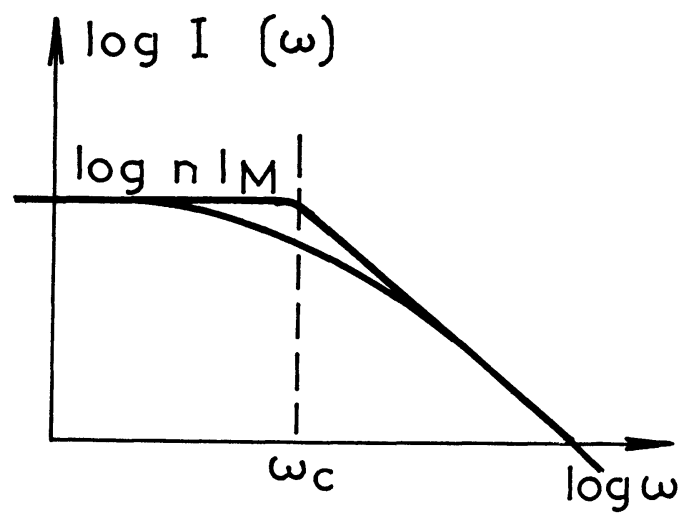

FIG. II.8. - Courant maximal $I(\omega)$.

II.4.2 Détermination de la puissance de sortie. Soit $k=\sqrt{L_{s} / L_{p}}$ le rapport de transformation du transformateur. Conformément à (I.7) et (II.1), nous avons :
$P_{\text {filtre }}=n \mathfrak{S}$ avec $\mathfrak{T}$ puissance relative à un transistor

$$
\mathfrak{T}=l_{p}\left[\omega \frac{I_{M}^{2}}{1+\frac{\omega^{2}}{\omega_{c}^{2}}}\right]_{M} .
$$

Le produit entre crochets est maximum pour $\omega=\omega_{c}$. Nous avons :

$$
\mathfrak{T}=\frac{\omega_{c}}{2} \cdot l_{p} I_{M}^{2} .
$$

II.4.3 Maximisation de la puissance de sortie. La position de la droite (2) du domaine de fonctionnement (cf. paragraphe II.2) étant fixée et la pulsation $\omega_{c}$ étant une fonction de $X, Y, l_{p}$ le problème est de savoir quelles valeurs prendre pour $X, Y, E$ afin que $\mathfrak{T}$ soit maximum pour un transistor donné.

On montre facilement que $\mathfrak{T}$ est maximum quand :

$$
E=\frac{V_{M}}{2} ; \quad X=Y=\frac{V_{M}}{2 I_{M}} .
$$

On a alors (cf. figure II.3) :

$$
\mathfrak{T}_{\max }=\frac{I_{M} V_{M}}{4}=\frac{W^{\prime}}{2} .
$$

La puissance maximum de l'étage est :

$$
P_{\text {filtre } \max }=\frac{n W^{\prime}}{2} .
$$

II.5 Dimensionnement de L'ÉtAge. - II.5.1 Calcul rigoureux. - Le dimensionnement consiste à déterminer le nombre de transistors $\mathrm{n}$ et le rapport de transformation $k$, en fonction des paramètres $E$, $I_{M}$ et $X$ qui dépendent du type de transistor choisi, et en fonction de l'ondulation résiduelle du redresseur. Définissons maintenant les ondulations en courant :

$-i_{R}(t)$ dans la charge en l'absence de filtrage.

$-i_{s}(t)$ dans l'inductance $L_{s}$ quand le filtrage agit.

On a :

$$
\frac{i_{s}(t)}{i_{R}(t)}=\frac{L_{\mathrm{ch}}}{L_{s}} .
$$

en supposant que la fréquence propre de la charge est faible par rapport à celle de l'ondulation $\bigcup_{R}(t)$ ce qui est pratiquement toujours le cas. La tension d'ondulation $\vartheta_{R}(t)$ ramenée au primaire donne une tension $\bigcup_{P}(t)$ qui doit être telle qu'elle n'entraîne pas la saturation des transistors de l'étage de puissance. D'après le paragraphe II.4.1, on doit avoir :

$$
|\vartheta(t)| \leqslant E
$$

(cf. Fig. II.9). D'où

$$
|\vartheta(t)|=\left|\frac{2 V_{R}(t)}{k}+\frac{X}{n} k \frac{L_{\mathrm{ch}}}{L_{s}} i_{R}(t)\right| \leqslant E .
$$

Si $\mho_{R}(t)$ et $i_{R}(t)$ sont des fonctions connues exactement 
on peut faire un calcul rigoureux. On obtient à l'aide de l'inéquation précédente une relation entre $n$ et $k$ et on rend $n$ minimum en fonction de $k$.

II.5.2 Calcul approché. - Si $\mho_{R}(t)$ et $i_{R}(t)$ ne sont pas exactement connues on peut cependant obtenir un certain nombre de relations générales comme nous allons le voir. Soient $\mathcal{V}_{R m}$ et $i_{R m}$ tels que

$$
\mho_{R m}=\left|\mho_{R}(t)\right|_{\max } \text { et } i_{R m}=\left|i_{R}(t)\right|_{\max } .
$$

Supposons que $\mho_{R}(t)$ et $i_{R}(t)$ prennent simultanément les valeurs $\mathcal{V}_{R m}$ et $i_{R m}$ (ou $-\mathcal{V}_{R m}$ et $-i_{R m}$ ), ce qui est évidemment le cas le plus défavorable. Nous obtenons à partir de la relation (II. 8) la relation (II.9) :

$$
2 \frac{\bigcup_{R m}}{k}+k \frac{X}{n} \frac{L_{\mathrm{ch}}}{L_{s}} i_{R m} \leqslant E .
$$

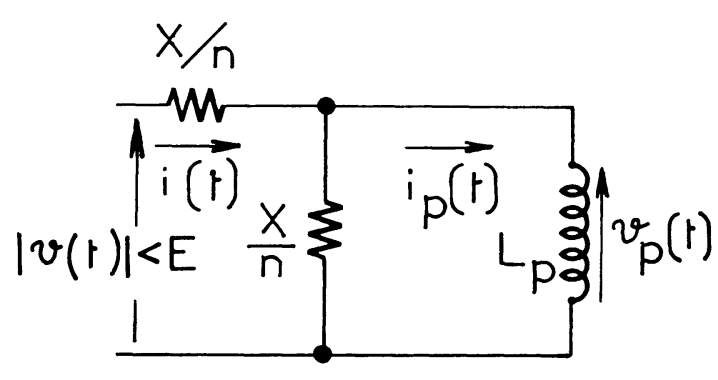

FIG. II.9. - Etage de puissance en fonctionnement limite.

On montre facilement que $\mathrm{n}$ est minimum pour :

$$
k=\frac{4 \mho_{R m}}{E} .
$$

On a alors :

$$
\omega_{c}=\frac{\vartheta_{R m}}{i_{R m} L_{\mathrm{ch}}}
$$

et

$$
P_{\text {filtre }}=2 \bigcup_{R m} i_{R m} \frac{L_{\mathrm{ch}}}{L_{s}} .
$$

Cette dernière expression est intéressante car $\mathcal{V}_{R m}$ et $i_{R m}$ sont facilement mesurables. Connaissant la puissance $\mathfrak{T}$ délivrée par un transistor (cf. par. II.4.3) on en déduit le nombre $\mathrm{n}$ de transistors de chaque moitié de l'étage de puissance.

Soient $\vartheta_{R 1}$ l'amplitude $\mathrm{du}$ fondamental de $\bigcup_{R}(t), i_{R 1}$ l'amplitude du fondamental de $i_{R}(t)$ et $\omega_{1}$ la pulsation correspondante, nous avons :

$$
i_{R 1}=\frac{\vartheta_{R 1}}{L_{\mathrm{ch}} \omega_{1}} \text {. }
$$

En considérant en première approximation que

$$
\frac{\vartheta_{R 1}}{i_{R 1}} \simeq \frac{\vartheta_{R m}}{i_{R m}}
$$

il vient :

$$
\omega_{c} \simeq \omega_{1}
$$

ce qui donne l'ordre de grandeur de $\omega_{c}$

II.5.3 Cas particuliers. Approximation en dent de scie de $\vartheta_{R}(t)$. - L'ondulation de la tension de sortie d'un redresseur polyphasé sans filtrage passif se rapproche en général d'une fonction en dent de scie.

$$
\vartheta_{R}(t)=V_{0}\left(1-\frac{2 t}{T}\right), \quad 0<t<T
$$

$\vartheta_{p}(t)$ et $i_{p}(t)$ (cf. Fig. II.9) sont alors comme sur la figure II. 10. Dans ce cas, il y a rigoureusement simultanéité de $\mathcal{V}_{R m}$ et $i_{R m}$ ce qui peut donner en pratique une grande importance aux formules précédentes.

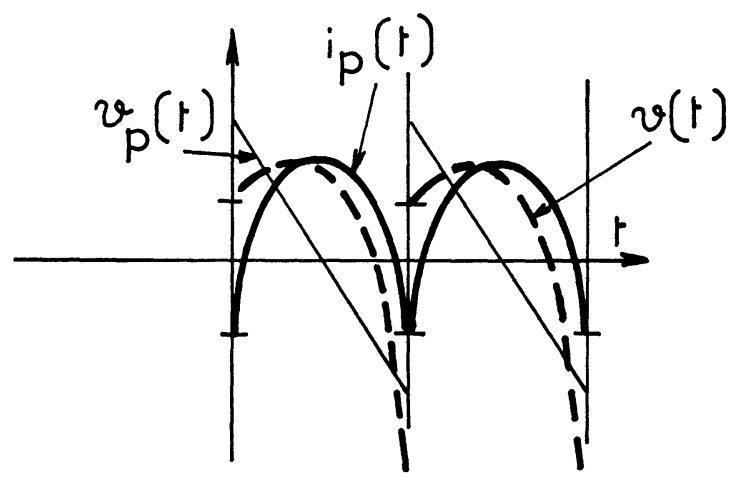

FIG. II.10. - Courant $i_{p}(t)$ dans le primaire du transformateur pour une ondulation en tension du redresseur en dent de scie.

11 vient alors

$$
\begin{gathered}
P_{\text {filtre }}=\frac{T V_{0}^{2}}{3 L_{s}} \quad \text { pour } \quad k=\frac{4 V_{0}}{E} \\
\omega_{c}=\frac{6}{T}=6 \frac{\omega_{1}}{2 \pi} \simeq \omega_{1} ; n=\frac{4}{3} \frac{T X}{L_{s}} \frac{V_{0}^{2}}{E^{2}} .
\end{gathered}
$$

Approximation sinusoïdale de $\mho_{R}(t)$. — L'ondulation de la tension de sortie d'un redresseur polyphasé, avec un fort filtrage passif, est sensiblement une sinusoïde $\vartheta_{R}=V_{0} \sin \omega_{1} t$.

Dans ce cas nous avons évidemment :

$$
\begin{gathered}
P_{\text {filtre }}=\frac{V_{0}^{2}}{L_{s} \omega_{1}} \quad \text { et } \quad k=\frac{4 V_{0}}{\sqrt{2} E}, \\
n=\frac{4 V_{0}^{2}}{E^{2}} \frac{X}{L_{s} \omega_{1}}, \quad \omega_{c}=\omega_{1} .
\end{gathered}
$$

Remarque I : Sous-harmoniques dans $V_{R}(t)$. - En général il existe dans le signal de sortie du redresseur une composante à basse fréquence (sous multiple de $\left.\omega_{1} / 2 \pi\right)$ due aux imperfections de ce redresseur. Cette composante est plus importante sur le courant $i_{R}(t)$ que sur la tension $\bigcup_{R}(t)$ par suite de l'effet de filtrage de l'impédance de la charge.

Si la connaissance de $\mathcal{V}_{R m}$ et $i_{R m}$ est consécutive à 
une mesure, les valeurs obtenues tiennent évidemment compte de cette ondulation.

$\mathrm{Si} \bigcup_{R m}$ et $i_{R m}$ résultent d'un calcul effectué à partir de l'ondulation théorique du redresseur, on obtient deux valeurs $\mho_{R m \text { th }}$ et $i_{R m \text { th }}$ n'en tenant pas compte. Il est alors nécessaire de connaître les amplitudes $\mathcal{V}_{p o}$ et $i_{p o}$ de l'ondulation basse fréquence, ce qui ne peut se faire qu'expérimentalement. La puissance du filtre est alors :

$$
P_{\text {filtre }}=2\left(\mathcal{V}_{R m \mathrm{th}}+\mathcal{V}_{p o}\right)\left(i_{R m \mathrm{th}}+i_{p o}\right) \frac{L_{\mathrm{ch}}}{L_{s}} .
$$

Lorsque le redresseur a un nombre de phases égal ou supérieur à 12 , on peut envisager la réduction de ses sous-harmoniques par l'installation d'une boucle locale [2] conformément à la figure II.11 qui résulte de la figure I.2. Il est bien évident que le gain $K$ considéré au paragraphe I. 3 doit alors être remplacé par la quantité $1 / \beta$. On peut également arriver au même résultat par un choix judicieux de la fréquence $f_{s}$ (cf. paragraphe I.3).

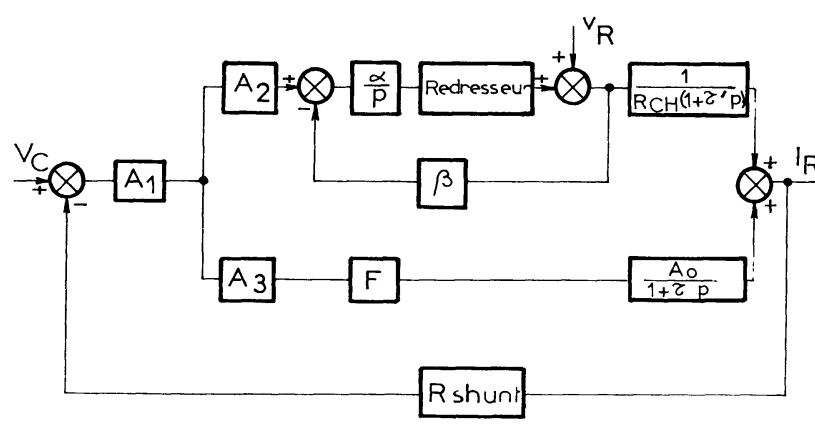

FIG. II.11. - Schéma bloc du système (avec bouclage local du redresseur)

La réduction des sous-harmoniques par le redresseur lui-même est très importante pour que la puissance du filtre actif soit minimale. Ceci est surtout vrai lorsqu'on utilise un filtre passif, les sous-harmoniques pouvant alors devenir prépondérants.

Remarque II : Choix de l'inductance. - La puissance du filtre $P_{\text {filtre }}$ et le nombre de transistors $n$ sont inversement proportionnels à $L_{s}$. Vis-à-vis de la puissance à installer, on a donc intérêt à ce que la valeur de $L_{s}$ soit la plus grande possible.

Toutefois, la valeur maximum de $L_{s}$ est fixée par l'un ou l'autre des deux aspects suivants :

- l'augmentation de la constante de temps de la charge totale du redresseur,

- l'énergie emmagasinée dans $L_{s}$ (cf. par. II.6).

Remarque III : Utilisation pratique des formules précédentes. - Il convient naturellement, en pratique, de prendre un coefficient de sécurité sur le nombre de transistors à utiliser et sur le rapport de transformation, de façon à tenir compte des approximations faites et d'effets parasites divers, en particulier l'inductance des câbles de liaison qui peut augmenter notablement la tension d'ondulation vue par les transistors.

II. 6 TRANSFORMATEUR DE SORTIE. - Le transformateur de sortie doit présenter les caractéristiques suivantes :

- un couplage très serré des enroulements afin d'éviter les flux de fuite,

- une symétrie aussi parfaite que possible des deux demi-primaires,

- des capacités parasites aussi faibles que possible,

- un volume de cuivre et un volume de fer raisonnables.

Le secondaire $L_{s}$ véhiculant le courant continu débité dans l'aimant, le dimensionnement du transformateur est conditionné par le secondaire.

En pratique, on rencontre des difficultés pour concilier toutes les exigences précitées. En effet, on stocke une grande énergie dans l'inductance secondaire $L_{s}$ qui est donc de grandes dimensions [1-2]. Le rapport de transformation $k$ est en général grand et il est très difficile dans ces conditions d'avoir un bon couplage. De plus, l'inductance primaire $L_{p}$ est alors faible et les inductances des câbles qui relient les transistors de puissance au transformateur prennent une grande importance. On est alors conduit au montage suivant (cf. Fig. II. 12).

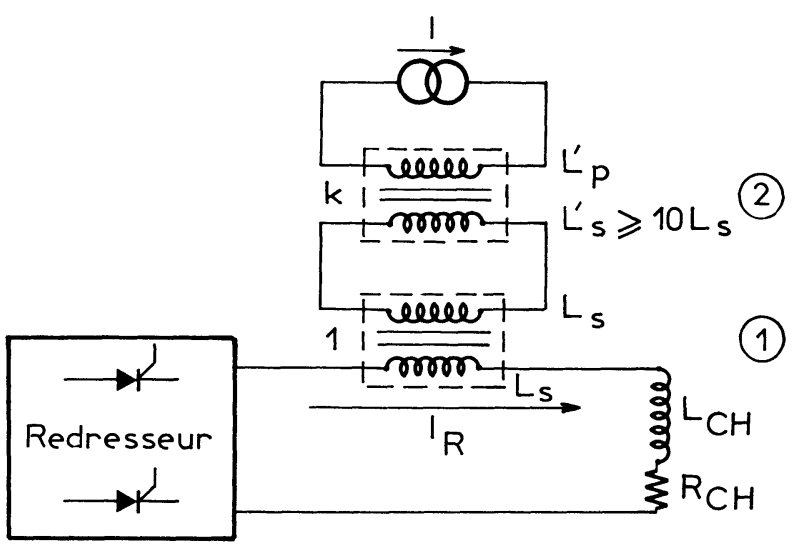

FIG. II.12. - Structure du transformateur de sortie.

L'avantage de cette solution est qu'on sépare la fonction «transformation » de la fonction "stockage d'énergie ». Le transformateur (1) est naturellement de grandes dimensions mais son rapport de transformation égal à 1 permet d'avoir un bon couplage. Le transformateur (2) qui n'est pas parcouru par le courant continu est de petites dimensions ce qui présente deux avantages :

- On peut lui donner sans difficulté la structure que l'on veut pour que le couplage soit bon malgré la valeur de son rapport de transformation $k$.

- On peut le disposer très près des transistors de puissance de façon à ce que les câbles de liaison soient situés dans un circuit haute impédance. 
Conclusion. - Notre étude se situe dans le cadre de la régulation d'impulsions de courant de longue durée produites par des générateurs utilisant un groupe redresseur polyphasé et un filtre actif, impulsions destinées en général à des aimants de grande puissance.

Les performances en ondulation résiduelle obtenues avec ces ensembles redresseurs et filtres actifs sont très variables suivant la grandeur considérée (tension ou courant) et suivant les caractéristiques de la charge. On peut dire que l'adjonction d'un filtre actif permet de gagner au minimum un facteur 100 sur les performances du système initial (redresseur, filtre passif, charge).

Sur le plan de la conception de l'organe de filtrage, nous avons proposé un étage de puissance à transistors dont la structure permet d'assurer la protection des transistors vis-à-vis des contraintes électriques, notamment vis-à-vis du phénomène de deuxième disruption.

Nous avons donné un certain nombre de formules générales permettant d'effectuer le dimensionnement de l'étage de puissance du filtre, même si on ne connaît pas en toute rigueur les caractéristiques de l'ondulation résiduelle du redresseur. Ces formules sont particulièrement intéressantes au stade d'un avant-projet.

Plus généralement, nous avons traité des principaux problèmes qui se posent lors de la synthèse d'un ensemble redresseur filtre actif en régime pulsé.

Nous espérons que ces résultats pourront rendre service à ceux qui se préoccupent de tels problèmes.

\section{Bibliographie}

[1] Lagasse (J.), Giralt (G.), Prajoux (R.), Spécification pour un projet de filtre dynamique standard. Rapport d'étude au CERN, Toulouse, 1968.

[2] JuANOLE (G.), Etude des filtres dynamiques destinés à la régulation d'impulsions de courant de très forte puissance. Thèse de Doctorat de $3^{\mathrm{e}}$ cycle, Université de Toulouse, Avril 1968.

[3] Domingues novo (D.), Contribution à l'étude de la deuxième disruption dans les transistors. Thèse de Doctorat ès Sciences Physiques, Université de Toulouse, décembre 1966.

[4] Corazza (M.), Etude expérimentale des phénomènes liés à la deuxième disruption dans les transistors plans au silicium. Thèse de Doctorat de $3^{\mathrm{e}}$ cycle, Université de Toulouse, décembre 1966.

[5] Scarlett (R. M.), Shockley (W.), Secondary Breakdown and hot spots in power transistors IEEE Int. Convention Record Part 3, March 25-28, 3-13.
[6] Prajoux (R.), Etablissement d'un modèle pour le comportement local d'un redresseur polyphasé utilisé en tant qu'amplificateur de puissance à réponse rapide. C. $R$. Acad. Sc., Paris, 1969, 262, 477.

[7] Prajoux (R.), Sevely (Y.), Thierry (P.), Transmittance en $z$ d'asservissements échantillonnés à signal d'erreur discontinu. Electronics Letters, 1968, 4, 242.

[8] Giraud (A.), Applications des récurrences à l'étude de certains systèmes de commande. Thèse de Docteur-Ingénieur, Toulouse le 21-4-69.

[9] IsCH (H. W.), Rapport C. E. R. N. 66-31. Proton Synchroton Machine Division, 26 octobre 1966.

[10] Toumire (E.), Etude et réalisation d'un générateur stabilisé d'impulsions rectangulaires de courant de très forte intensité. Thèse de Docteur-Ingénieur, Toulouse le 6 juin 1966. 